Publications of the Astronomical Society of the Pacific, 113:622-638, 2001 May

(C) 2001. The Astronomical Society of the Pacific. All rights reserved. Printed in U.S.A.

\title{
Polarizing Grids, Their Assemblies, and Beams of Radiation
}

\author{
Martin Houde, ${ }^{1,2}$ Rachel L. Akeson,${ }^{3}$ John E. Carlstrom,${ }^{4}$ James W. Lamb,${ }^{5}$ \\ David A. Schleuning, ${ }^{4}$ And David P. Woody ${ }^{5}$ \\ Received 2000 October 27; accepted 2001 February 5
}

\begin{abstract}
This paper gives an analysis of the behavior of polarizing grids and reflecting polarizers by solving Maxwell's equations, for arbitrary angles of incidence and grid rotation, for cases where the excitation is provided by an incident plane wave or a beam of radiation. The scattering and impedance matrix representations are derived and used to solve more complicated configurations of grid assemblies. The results are also compared with data obtained in the calibration of reflecting polarizers at the Owens Valley Radio Observatory. From this analysis, we propose a method for choosing the optimum grid parameters (wire radius and spacing). We also provide a study of the effects of two types of errors (in wire separation and radius size) that can be introduced in the fabrication of a grid.
\end{abstract}

\section{INTRODUCTION}

The literature on wire grids is abundant, and they have been studied with different techniques and for numerous applications. Most of the analyses were, however, restricted to special cases of incident field and grid orientations. The more general and arbitrary situation seems to have been first studied by Wait (see Wait 1955 and Larsen 1962). This problem is addressed again in this paper and follows a line of analysis fairly similar to the one used by Wait. Our treatment is, however, more general in that we do not assume that the wires of the grid are induced with only a longitudinal current; we will indeed show that an azimuthal component is also present. We also solve for the induced current by considering the tangential components of both the electric and magnetic fields at the surface of the wires.

This analysis is carried out in the next two sections and will serve as the basis for our treatment of the reflecting polarizer (§ 4) and the introduction of the scattering and impedance matrix representations for a grid $(\S 3.3)$, which will in turn enable us to briefly discuss more complicated systems. These matrices will be particularly useful in allowing us to define what will be called the principal axes of a grid. These are two orthogonal and independent directions of polarization in the

\footnotetext{
${ }^{1}$ Caltech Submillimeter Observatory, 111 Nowelo Street, Hilo, HI 96720; houde@ulu.submm.caltech.edu.

${ }^{2}$ Département de Physique, Université de Montréal, Montréal, QC H3C 3J7, Canada.

${ }^{3}$ Infrared Processing and Analysis Center, California Institute of Technology, Pasadena, CA 91125.

${ }^{4}$ Department of Astronomy and Astrophysics and Enrico Fermi Institute, University of Chicago, Chicago, IL 60637.

${ }^{5}$ Owens Valley Radio Observatory, California Institute of Technology, Big Pine, CA 93513.
}

plane of the incident radiation along which an arbitrary electric field can be decomposed and shown to scatter without crosspolarization. With this representation at hand, it will then be possible to derive a set of optimal parameters (wire radius and spacing) to be used in the selection of a grid. We will also present an analysis of the effects of random errors that can be introduced in the fabrication of grids, the results obtained will then be compared to experimental results previously published by Shapiro \& Bloemhof (1990).

The last section will be dedicated to the study of the more subtle impacts that the nature of the incoming radiation can have on the response of a grid assembly such as a reflecting polarizer ( $\$ 4)$. Although limited to this particular case, our discussion could possibly apply to other types of instruments. We have also included (Appendix B) a list of the symbols used in the different equations.

\section{THE CASE OF A SINGLE WIRE}

Before trying to solve the problem of the grid or the reflecting polarizer, it is preferable to study the case of a single conducting wire. It will serve as the basis for our studies of the more complicated cases to follow in subsequent sections.

Let us suppose that a wire of radius $a$ is oriented, as depicted in Figure 1, parallel to the $x$-axis at $y=y_{0}, z=z_{0}$ and that it is subjected to an incident plane wave $\boldsymbol{E}_{i}(\boldsymbol{r})$ of arbitrary direction and polarization:

$$
\boldsymbol{E}_{i}(\boldsymbol{r})=E_{0}\left(\alpha^{\prime} \boldsymbol{e}_{x}+\beta^{\prime} \boldsymbol{e}_{y}+\gamma^{\prime} \boldsymbol{e}_{z}\right) \exp [-j(\boldsymbol{k} \cdot \boldsymbol{r}-\omega t)]
$$

with

$$
\boldsymbol{k}=k\left(\alpha \boldsymbol{e}_{x}+\beta \boldsymbol{e}_{y}+\gamma \boldsymbol{e}_{z}\right)
$$

and where, of course, the following conditions of normalization 


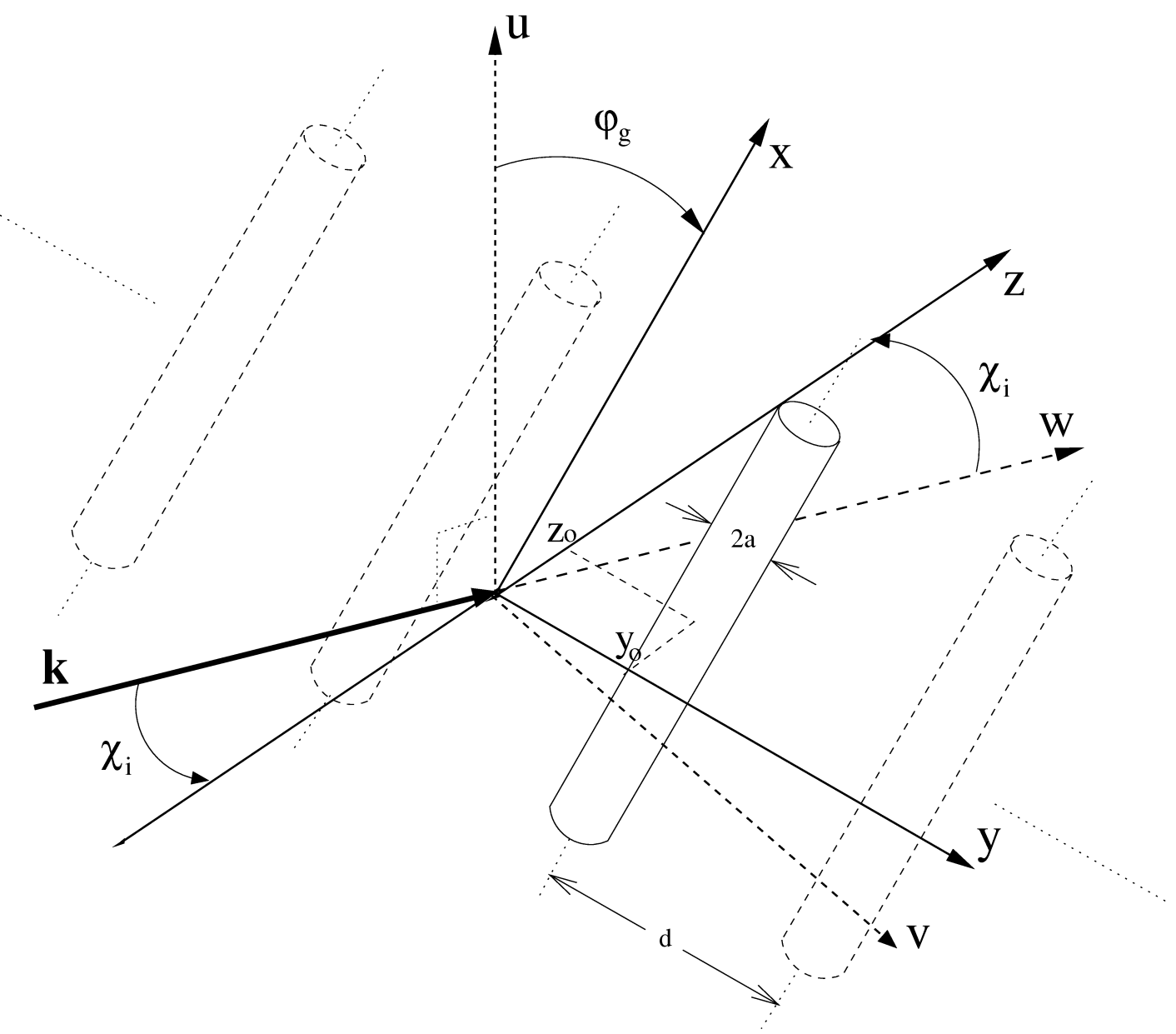

FIG. 1.-Coordinates system for the study of a polarizing grid or a single wire. The wavevector $\boldsymbol{k}$ of the incident radiation is aligned with the $w$-axis, the $u$-, $x$-, and $y$-axes are in the plane of the page, the $w$-and $z$-axes are in the plane perpendicular to the $u$-axis (into the page), and the wires are parallel to the $x y$ plane. We refer to the $(u, v, w)$ and $(x, y, z)$ systems as the laboratory and grid coordinates, respectively.

and orthogonality apply:

$$
\begin{gathered}
\alpha^{2}+\beta^{2}+\gamma^{2}=\alpha^{\prime 2}+\beta^{\prime 2}+\gamma^{\prime 2}=1, \\
\alpha \alpha^{\prime}+\beta \beta^{\prime}+\gamma \gamma^{\prime}=0 .
\end{gathered}
$$

Using the coordinate system depicted in Figure 1, we have

$$
\begin{aligned}
& \alpha=\sin \left(\chi_{i}\right) \sin \left(\varphi_{g}\right), \\
& \beta=\sin \left(\chi_{i}\right) \cos \left(\varphi_{g}\right), \\
& \gamma=\cos \left(\chi_{i}\right),
\end{aligned}
$$

where $\chi_{i}$ is the angle of incidence and $\varphi_{g}$ is the angle of grid rotation.

In everything that follows, we will drop the $\exp (j \omega t)$ term and assume it to be implicit in the equations. We will also suppose that the wire is of infinite length and made of a good conducting material of conductivity $\sigma$ such that any current flowing through it can be accurately represented by a surface current vector $\boldsymbol{K}$. This quantity is related to the current density $\boldsymbol{J}(\boldsymbol{r})$ as follows:

$$
\boldsymbol{J}(\boldsymbol{r})=\boldsymbol{K} \delta(\rho-a) \exp (-j \boldsymbol{k} \cdot \boldsymbol{r}),
$$

where

$$
\boldsymbol{K}=K^{x} \boldsymbol{e}_{x}+K^{\theta} \boldsymbol{e}_{\theta}
$$

and $y-y_{0}=\rho \cos (\theta), z-z_{0}=\rho \sin (\theta)$.

Before we solve for the scattered fields, it is to our advantage to note that for the case considered here (i.e., thin wire with an approximate solution involving no angular mode dependency), the problem can be broken in two parts or modes. The mode where the electrical field is parallel to the plane defined by $\boldsymbol{e}_{x}$ and $\boldsymbol{k}$ (the transverse magnetic or TM- 
mode) is related to the presence of $K^{x}$, while the mode where the magnetic field is parallel to this same plane, the transverse electric or TE-mode, is related to $K^{\theta}$. The analysis will, therefore, be facilitated with the use of the two vector potentials $\boldsymbol{A}_{s}$ and $\boldsymbol{F}_{s}$ for the scattered fields (Balanis 1989).

The TM-mode can be analyzed using the vector potential $\boldsymbol{A}_{s}$, in the Lorentz gauge, with $\boldsymbol{F}_{s}=0$. The needed equations are

$$
\begin{aligned}
& \boldsymbol{A}_{s}(\boldsymbol{r})=\frac{\mu_{0}}{4 \pi} \int \boldsymbol{J}\left(\boldsymbol{r}^{\prime}\right) \frac{\exp (-j k R)}{R} d^{3} r^{\prime}, \\
& \boldsymbol{E}_{s}(\boldsymbol{r})=\frac{c^{2}}{j \omega} \nabla\left(\boldsymbol{\nabla} \cdot \boldsymbol{A}_{s}(\boldsymbol{r})\right)-j \omega \boldsymbol{A}_{s}(\boldsymbol{r}), \\
& \boldsymbol{H}_{s}(\boldsymbol{r})=\frac{1}{\mu_{0}} \boldsymbol{\nabla} \times \boldsymbol{A}_{s}(\boldsymbol{r})
\end{aligned}
$$

with

$$
R^{2}=\left(x-x^{\prime}\right)^{2}+\left(y-y^{\prime}\right)^{2}+\left(z-z^{\prime}\right)^{2} .
$$

Since we are concerned here with the longitudinal component of the surface current density, we need to consider only the $A^{x}$ component of the vector potential (i.e., we set $A^{\rho}=$ $A^{\theta}=0$ ). Equation (3) can be solved exactly when $\boldsymbol{K}$ is expanded with a Fourier series, but in cases where the wavelength of the incident wave is much larger than the wire radius, it can be shown that

$$
A_{s}^{x}(\boldsymbol{r})=\frac{\pi \mu_{0} a}{2 j} K^{x} H_{0}^{(2)}\left(k^{\prime} \rho\right) \exp (-j \varphi)
$$

with $k^{\prime}=k \sqrt{1-\alpha^{2}}$ and $\varphi=k\left(\alpha x+\beta y_{0}+\gamma z_{0}\right)$ and where $H_{n}^{(2)}(x)$ is Hankel's function of the second kind of order $n$.

On the other hand, it is advantageous to study the TE-mode with the vector potential $\boldsymbol{F}_{s}$, in the appropriate gauge, with $\boldsymbol{A}_{s}=0$ (Balanis 1989). To do so, we will not consider the effect of the current density (more precisely, its azimuthal component) but rather that of the magnetization vector $\boldsymbol{M}$ that it induces. The relevant equations are now

$$
\begin{aligned}
\boldsymbol{J}(\boldsymbol{r}) & =\boldsymbol{\nabla} \times \boldsymbol{M}(\boldsymbol{r}), \\
\boldsymbol{F}_{s}(\boldsymbol{r}) & =\frac{j \omega \mu_{0} \varepsilon_{0}}{4 \pi} \int \boldsymbol{M}\left(\boldsymbol{r}^{\prime}\right) \frac{\exp (-j k R)}{R} d^{3} r^{\prime}, \\
\boldsymbol{E}_{s}(\boldsymbol{r}) & =-\frac{1}{\varepsilon_{0}} \boldsymbol{\nabla} \times \boldsymbol{F}_{s}(\boldsymbol{r}), \\
\boldsymbol{H}_{s}(\boldsymbol{r}) & =\frac{c^{2}}{j \omega} \boldsymbol{\nabla}\left(\boldsymbol{\nabla} \cdot \boldsymbol{F}_{s}(\boldsymbol{r})\right)-j \omega \boldsymbol{F}_{s}(\boldsymbol{r})
\end{aligned}
$$

with $R$ as defined above.

Since we are now concerned with the azimuthal component of the surface current density, we need to consider only the $M^{x}$ and $F_{s}^{x}$ components of the magnetization and vector potential (i.e., we have $M^{\rho}=M^{\theta}=F_{s}^{\rho}=F_{s}^{\theta}=0$ ). Again, in cases where the wavelength of the incident wave is much larger than the wire radius, it can be shown that

$$
F_{s}^{x}(\boldsymbol{r})=\frac{\pi \omega \mu_{0} \varepsilon_{0} a^{2}}{4} K^{\theta} H_{0}^{(2)}\left(k^{\prime} \rho\right) \exp (-j \varphi) .
$$

It is now straightforward to calculate the scattered fields by combining the solution obtained for each mode (using eqs. [4], [5], and [6] for the TM-mode and eqs. [9], [10], and [11] for the TE-mode):

$$
\begin{aligned}
& E_{s}^{\rho}(\boldsymbol{r})=-j \alpha \sqrt{1-\alpha^{2}} F K^{x} H_{1}^{(2)}\left(k^{\prime} \rho\right) \exp (-j \varphi), \\
& E_{s}^{\theta}(\boldsymbol{r})=-\sqrt{1-\alpha^{2}} F \frac{k a}{2} K^{\theta} H_{1}^{(2)}\left(k^{\prime} \rho\right) \exp (-j \varphi), \\
& E_{s}^{x}(\boldsymbol{r})=-\left(1-\alpha^{2}\right) F K^{x} H_{0}^{(2)}\left(k^{\prime} \rho\right) \exp (-j \varphi), \\
& H_{s}^{\rho}(\boldsymbol{r})=\frac{\alpha \sqrt{1-\alpha^{2}}}{Z_{0}} F \frac{k a}{2} K^{\theta} H_{1}^{(2)}\left(k^{\prime} \rho\right) \exp (-j \varphi), \\
& H_{s}^{\theta}(\boldsymbol{r})=-j \frac{\sqrt{1-\alpha^{2}}}{Z_{0}} F K^{x} H_{1}^{(2)}\left(k^{\prime} \rho\right) \exp (-j \varphi), \\
& H_{s}^{x}(\boldsymbol{r})=-j \frac{\left(1-\alpha^{2}\right)}{Z_{0}} F \frac{k a}{2} K^{\theta} H_{0}^{(2)}\left(k^{\prime} \rho\right) \exp (-j \varphi),
\end{aligned}
$$

where $F=\left(\pi \mu_{0} \omega a\right) / 2$ and $Z_{0}=\sqrt{\mu_{0} / \varepsilon_{0}}$ is the impedance of free space. Note that although equations (12)-(17) represent the scattered field, the components of surface current density that are included in these equations are that of the, yet undetermined, total surface current density that we are now in a position to evaluate.

In order to do so, we must first express the incident plane wave in the appropriate coordinate system. This can be done by first using the following expression:

$$
\exp [-j k(\beta y+\gamma z)]=\sum_{n=-\infty}^{\infty}(-j)^{n} J_{n}\left(k^{\prime} \rho\right) \exp \left(j n \theta^{\prime}\right)
$$

with $\theta^{\prime}=\theta-\arctan (\gamma / \beta), J_{n}(x)$ the Bessel function of order $n$, and by again splitting the incident field in the two modes defined earlier (van de Hulst 1957, pp. 119-121, 297-301; Balanis 1989). This enables us to express the plane wave in cylindrical coordinates and match the fields with the usual boundary conditions for their tangential components at the surface of the wire. For the TM-mode the condition is

$$
E_{i}^{x}+E_{s}^{x}=Z_{s}\left(H_{i}^{\theta}+H_{s}^{\theta}\right)
$$


Within the order of precision used for our analysis $(\lambda \gg a)$ and considering a solution with no angular dependency, it can be shown that

$$
\begin{aligned}
E_{i}^{x} & \simeq \alpha^{\prime} E_{0} \exp \left[-j k\left(\alpha x+\gamma z_{0}\right)\right] \\
H_{i}^{\theta} & \simeq j \alpha^{\prime} \frac{E_{0}}{Z_{0}} \frac{k a}{2} \exp \left[-j k\left(\alpha x+\gamma z_{0}\right)\right] .
\end{aligned}
$$

For the TE-mode we have

$$
E_{i}^{\theta}+E_{s}^{\theta}=-Z_{s}\left(H_{i}^{x}+H_{s}^{x}\right)
$$

with

$$
\begin{aligned}
& E_{i}^{\theta} \simeq-j\left(\gamma^{\prime} \beta-\beta^{\prime} \gamma\right) E_{0} \frac{k a}{2} \exp \left[-j k\left(\alpha x+\gamma z_{0}\right)\right] \\
& H_{i}^{x} \simeq\left(\gamma^{\prime} \beta-\beta^{\prime} \gamma\right) \frac{E_{0}}{Z_{0}} \exp \left[-j k\left(\alpha x+\gamma z_{0}\right)\right] .
\end{aligned}
$$

In equations (19) and (20), $Z_{s}=(1+j) \sqrt{\mu_{0} \omega / 2 \sigma}$ is the surface impedance of the wire (Jackson 1962). It is to be noted that for wires of small radius, relative to the wavelength, the boundary conditions (19) and (20) along with the equation for $Z_{s}$ represent approximations that are valid only in the lowest mode and for a sufficiently good conductor. A more rigorous treatment shows that these equations will be modified in the more general case (Wait 1979; Bouche, Molinet, \& Mittra 1997). But for the purpose of our analysis, the approximation used here is adequate.

When solving these two sets of equations, we find the following expressions for the components of the total surface current densities:

$$
\begin{aligned}
K^{x} & =\frac{E_{0}}{F} \frac{\alpha^{\prime}\left[1-j\left(Z_{s} / Z_{0}\right)(k a / 2)\right]}{\left(1-\alpha^{2}\right) H_{0}^{(2)}\left(k^{\prime} a\right)-j\left(Z_{s} / Z_{0}\right) \sqrt{1-\alpha^{2}} H_{1}^{(2)}\left(k^{\prime} a\right)}, \\
K^{\theta} & =\frac{E_{0}}{F} \frac{-j\left(\gamma^{\prime} \beta-\beta^{\prime} \gamma\right)\left[1+j\left(Z_{s} / Z_{0}\right)(2 / k a)\right]}{\sqrt{1-\alpha^{2}} H_{1}^{(2)}\left(k^{\prime} a\right)+j\left(Z_{s} / Z_{0}\right)\left(1-\alpha^{2}\right) H_{0}^{(2)}\left(k^{\prime} \rho\right)} .
\end{aligned}
$$

These last two equations can be inserted in equations (12)-(17) to calculate the value of the fields at any point exterior to the wire. For a good conductor the internal fields are practically nonexistent. Equations (21) and (22) are in agreement with the results presented in Balanis (1989, chap. 11) for the case of normal incidence and a perfectly conducting wire.

\section{THE POLARIZING GRID}

\subsection{Analysis}

With the solution for a single wire in hand, the problem of the configuration of an infinite number of wires of infinite length separated by a distance $d$ is simplified if one realizes that every wire will be induced with the same surface current $\boldsymbol{K}$. The only difference will be a phase term in the current density $\boldsymbol{J}(\boldsymbol{r})$, given by equation (2), which depends on the position of the wire along the $y$-axis. The same thing can be said for the scattered fields from any given wire; one only has to replace $y_{0}$ by $n d$ in equations (12)-(17), where $n$ is an integer that determines the position of the wire.

If the scattered fields are now just the sum of all the different scattered fields from the individual wires, care must however be taken in evaluating the surface current. First, when one matches the boundary conditions, it must be done simultaneously at the surface of every wire. However, since we are dealing with an infinite number of infinitely long wires subjected to the same incident plane wave, it turns out that it is sufficient to do so for only one of the wires. If the boundary conditions are matched for one wire, they will be for all. We have chosen for our calculations the "center" wire at $n=0$. Second, to match the boundary conditions we must express the scattered fields of each and every wire in a cylindrical coordinate system centered on the position of this "center" wire.

When this is done, we find the following expressions for the components of the induced total surface current density:

$$
\begin{aligned}
K^{x} & =\frac{E_{0}}{F} \alpha^{\prime} \frac{N_{x}}{\Delta_{x}}, \\
K^{\theta} & =-j \frac{E_{0}}{F}\left(\gamma^{\prime} \beta-\beta^{\prime} \gamma\right) \frac{N_{\theta}}{\Delta_{\theta}}
\end{aligned}
$$

with

$$
\begin{aligned}
& N_{x}=1-j \frac{Z_{s}}{Z_{0}} \frac{k a}{2}, \\
& \Delta_{x}=\left(1-\alpha^{2}\right) S_{1}-j \frac{Z_{s}}{Z_{0}} \sqrt{1-\alpha^{2}} H_{1}^{(2)}\left(k^{\prime} a\right), \\
& N_{\theta}=1+j \frac{Z_{s}}{Z_{0}} \frac{2}{k a}, \\
& \Delta_{\theta}=\sqrt{1-\alpha^{2}} H_{1}^{(2)}\left(k^{\prime} a\right)+j \frac{Z_{s}}{Z_{0}}\left(1-\alpha^{2}\right) S_{1},
\end{aligned}
$$

and

$$
S_{1}=H_{0}^{(2)}\left(k^{\prime} a\right)+2 \sum_{n=1}^{\infty} H_{0}^{(2)}\left(k^{\prime} n d\right) \cos (k \beta n d) .
$$


We will give in $\S 3.4$ adequate approximations for $\Delta_{x}$ and $\Delta_{\theta}$ that will greatly simplify the evaluation of the reflection and transmission coefficients that will soon follow.

By using the appropriate expansions for series of Hankel's functions, we can write the expressions for the components of the total electric field far away from the grid as

$$
\begin{aligned}
E_{T}^{x}(\boldsymbol{r})= & \alpha^{\prime} E_{0} \exp (-j \boldsymbol{k} \cdot \boldsymbol{r}) \\
& -\frac{\left(1-\alpha^{2}\right)}{\gamma} \frac{\lambda F}{\pi d} K^{x} \\
& \times \exp \left(-j k \gamma\left|z-z_{0}\right|\right) \exp (-j \varphi), \\
E_{T}^{y}(\boldsymbol{r})= & \beta^{\prime} E_{0} \exp (-j \boldsymbol{k} \cdot \boldsymbol{r}) \\
& +\frac{\lambda F}{\pi d}\left[\frac{\alpha \beta}{\gamma} K^{x}+j \frac{k a}{2} K^{\theta} \frac{z-z_{0}}{\left|z-z_{0}\right|}\right] \\
& \times \exp \left(-j k \gamma\left|z-z_{0}\right|\right) \exp (-j \varphi), \\
E_{T}^{z}(\boldsymbol{r})= & \gamma^{\prime} E_{0} \exp (-j \boldsymbol{k} \cdot \boldsymbol{r}) \\
& +\frac{\lambda F}{\pi d}\left[\alpha K^{x} \frac{z-z_{0}}{\left|z-z_{0}\right|}-j \frac{\beta}{\gamma} \frac{k a}{2} K^{\theta}\right] \\
& \times \exp \left(-j k \gamma\left|z-z_{0}\right|\right) \exp (-j \varphi),
\end{aligned}
$$

where $\varphi=k\left(\alpha x+\beta y+\gamma z_{0}\right)$. From these it is now straightforward to get the reflection and transmission coefficients (normalized to $E_{0}$ ) in the far field:

$$
\begin{aligned}
R^{x} & =-\frac{F}{E_{0}} \frac{\lambda}{\pi d} \frac{\left(1-\alpha^{2}\right)}{\gamma} K^{x}, \\
R^{y} & =\frac{F}{E_{0}} \frac{\lambda}{\pi d}\left[\frac{\alpha \beta}{\gamma} K^{x}-j \frac{k a}{2} K^{\theta}\right], \\
R^{z} & =-\frac{F}{E_{0}} \frac{\lambda}{\pi d}\left[\alpha K^{x}+j \frac{\beta}{\gamma} \frac{k a}{2} K^{\theta}\right], \\
T^{x} & =\alpha^{\prime}+R^{x}, \\
T^{y} & =\beta^{\prime}+\frac{F}{E_{0}} \frac{\lambda}{\pi d}\left[\frac{\alpha \beta}{\gamma} K^{x}+j \frac{k a}{2} K^{\theta}\right], \\
T^{z} & =\gamma^{\prime}+\frac{F}{E_{0}} \frac{\lambda}{\pi d}\left[\alpha K^{x}-j \frac{\beta}{\gamma} \frac{k a}{2} K^{\theta}\right],
\end{aligned}
$$

where we have set $z_{0}=0$ for simplicity.

Equations (30)-(35) along with equations (23) and (24) are the solution to the polarizing grid problem for cases where it is assumed that $k^{\prime} a \ll 1$ and $a \ll d$.

For predictions of measurements made in the laboratory, one merely has to transform these coefficients to the laboratory coordinate system. If we adopt for this system the coordinates of the incident/transmitted $(u, v, w)$ and reflected $\left(u^{\prime}, v^{\prime}, w^{\prime}\right)$ plane waves defined in Figures 1 and 2, the last system of equations is simplified to

$$
\begin{aligned}
R^{u^{\prime}} & =-\frac{F}{E_{0}} \frac{\lambda}{\pi d} \frac{1}{\gamma \sqrt{1-\gamma^{2}}}\left[\beta K^{x}-j \alpha \gamma \frac{k a}{2} K^{\theta}\right], \\
R^{v^{\prime}} & =-\frac{F}{E_{0}} \frac{\lambda}{\pi d} \frac{1}{\gamma \sqrt{1-\gamma^{2}}}\left[\alpha \gamma K^{x}+j \beta \frac{k a}{2} K^{\theta}\right], \\
R^{w^{\prime}} & =0, \\
T^{u} & =\alpha^{\prime \prime}-\frac{F}{E_{0}} \frac{\lambda}{\pi d} \frac{1}{\gamma \sqrt{1-\gamma^{2}}}\left[\beta K^{x}+j \alpha \gamma \frac{k a}{2} K^{\theta}\right], \\
T^{v} & =\beta^{\prime \prime}-\frac{F}{E_{0}} \frac{\lambda}{\pi d} \frac{1}{\gamma \sqrt{1-\gamma^{2}}}\left[\alpha \gamma K^{x}-j \beta \frac{k a}{2} K^{\theta}\right], \\
T^{w} & =0
\end{aligned}
$$

with $\alpha^{\prime \prime}$ and $\beta^{\prime \prime}$ related to the incident field by

$$
\boldsymbol{E}_{i}(\boldsymbol{r})=E_{0}\left(\alpha^{\prime \prime} \boldsymbol{e}_{u}+\beta^{\prime \prime} \boldsymbol{e}_{v}\right) \exp (-j k w) .
$$

As can be seen, the reflected and transmitted fields have no component along their respective direction of propagation as is required for the propagation of plane waves in free space.

\subsection{Effects of Grid Imperfections}

So far we have assumed that there were no imperfections in the construction of the grid; obviously (and unfortunately) such is not the case in a realistic situation. It would be instructive if we could calculate the effects of errors that are likely to be introduced in the fabrication process. In this section we will provide expressions that will allow us to evaluate changes in the reflection and transmission coefficients induced by two possible imperfections: random errors in wire spacing and random variations in the size of the wire radius.

\subsubsection{Random Errors in Wire Spacing}

It is our experience that some of the commercially available grids when observed under a microscope show some defects in their assembly. Visually, the most obvious manifestation of this is inconsistency in the spacing between wires. In order to calculate the effect of these errors, we have to go back to the discussion of $\S 3.1$ that guided us into the evaluation of the induced current on the wires. Since we can no longer assume that the wires are evenly spaced, we must now realize that they will in general have different values for the current and fields on their surface. This will be made more apparent if we write down the expression for the $x$-component of the electric field 


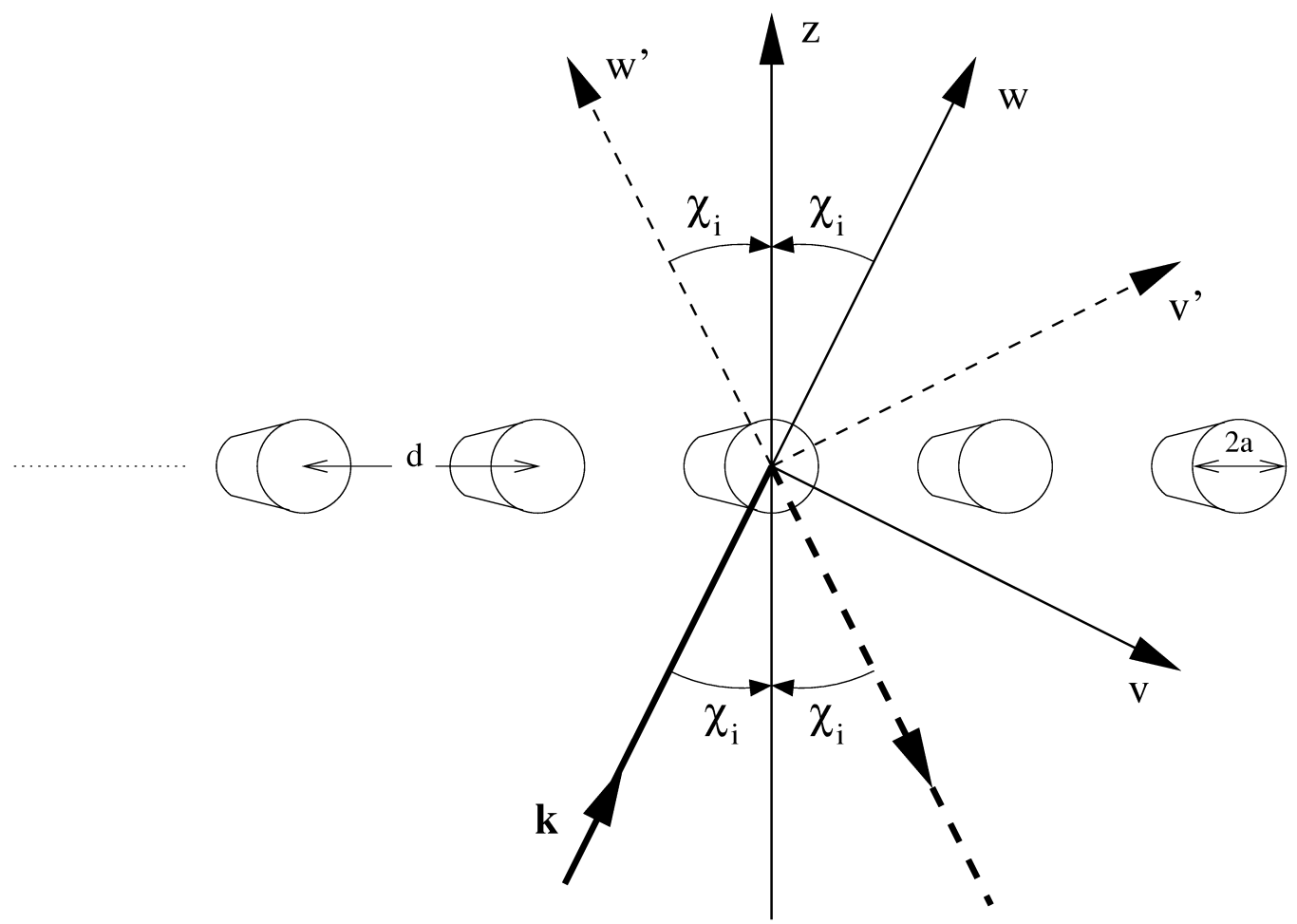

FIG. 2.-Definition of the system of coordinates $\left(u^{\prime}, v^{\prime}, w^{\prime}\right)$ for the reflected wave in relation to the $(u, v, w)$ system of the incident/transmitted waves introduced earlier in Fig. 1. The $u^{\prime}$ - and $u$-axes are one and the same and are pointing out of the page. The direction of propagation of the reflected wave is along the negative $w^{\prime}$-axis.

on the surface of the "center" wire:

$$
\begin{aligned}
E_{g}^{x}(a)= & -\left(1-\alpha^{2}\right) F \exp \left[-j k\left(\alpha x+\gamma z_{0}\right)\right] \\
& \times \sum_{n=-\infty}^{\infty} K_{n}^{x}(\xi) G_{n}(\xi)
\end{aligned}
$$

with

$$
G_{n}(\xi)= \begin{cases}H_{0}^{(2)}\left(k^{\prime} a\right) \exp \left(-j k \beta \xi_{0}\right), & n=0, \\ H_{0}^{(2)}\left(k^{\prime}\left|n d+\xi_{n}\right|\right) \exp \left[-j k \beta\left(n d+\xi_{n}\right)\right], & n \neq 0,\end{cases}
$$

where $K_{n}^{x}$ is the induced surface current on wire $n$ and the $\xi_{n}$ terms are statistically independent random errors in the positioning of the wires. Now, if $E\{x\}$ stands for the expected value of $x$ and if we suppose that the errors have a zero mean, we can write

$$
\begin{aligned}
E\left\{\xi_{m}^{r}\right\} & =0, \quad r=1,3,5 \ldots, \\
E\left\{\xi_{m}^{r}\right\} & =E\left\{\xi_{n}^{r}\right\}=E\left\{\xi^{r}\right\}, \quad \forall m, n, \\
E\left\{\xi_{m}^{r} \xi_{n}^{s}\right\} & =E\left\{\xi_{m}^{r}\right\} E\left\{\xi_{n}^{s}\right\}, \quad m \neq n, \\
\overline{\xi^{m} K^{x}} & =\overline{\xi_{n}^{m} K_{n}^{x}}=E\left\{\xi_{n}^{m} K_{n}^{x}\right\}, \quad \forall m, n .
\end{aligned}
$$

The first equation is deduced from the supposed evenness of the probability density function of the errors, the second states that their statistics are the same across the grid and the third expresses their statistical independence. The last of these equations arises from the fact that if we were to test a large number of similar grids, every wire would exhibit the same average value $\overline{\xi^{m} K^{x}}$ for any induced surface current moment (independent of its position $n$ ).

We will not go into the details of the calculations as they are somewhat lengthy, but it can be shown that if we apply this last set of equations and expand $K_{n}^{x}(\xi)$ and $G_{n}(\xi)$ with their Taylor series around $\xi_{m}=0$ while solving for the boundary conditions, we can find an expression (valid to the second order in $\xi$ ) for the average longitudinal surface current:

$$
\overline{K^{x}} \simeq K^{x}\left[1-\frac{E\left\{\xi^{2}\right\}}{\sum_{n=-\infty}^{\infty} G_{n}(0)} \sum_{m=-\infty}^{\infty}\left\{\frac{1}{2} \frac{\partial^{2} G_{m}}{\partial \xi_{m}^{2}}-\frac{\left(1-\alpha^{2}\right)}{\Delta_{x}}\left[\frac{\partial G_{m}}{\partial \xi_{m}}\right]^{2}\right\}_{\xi_{m}=0}\right]
$$

where $K^{x}$ is the current density induced on the wires of a perfect grid and is given by equation (23). One sees that the errors bring a perturbation that is proportional to their common variance. 


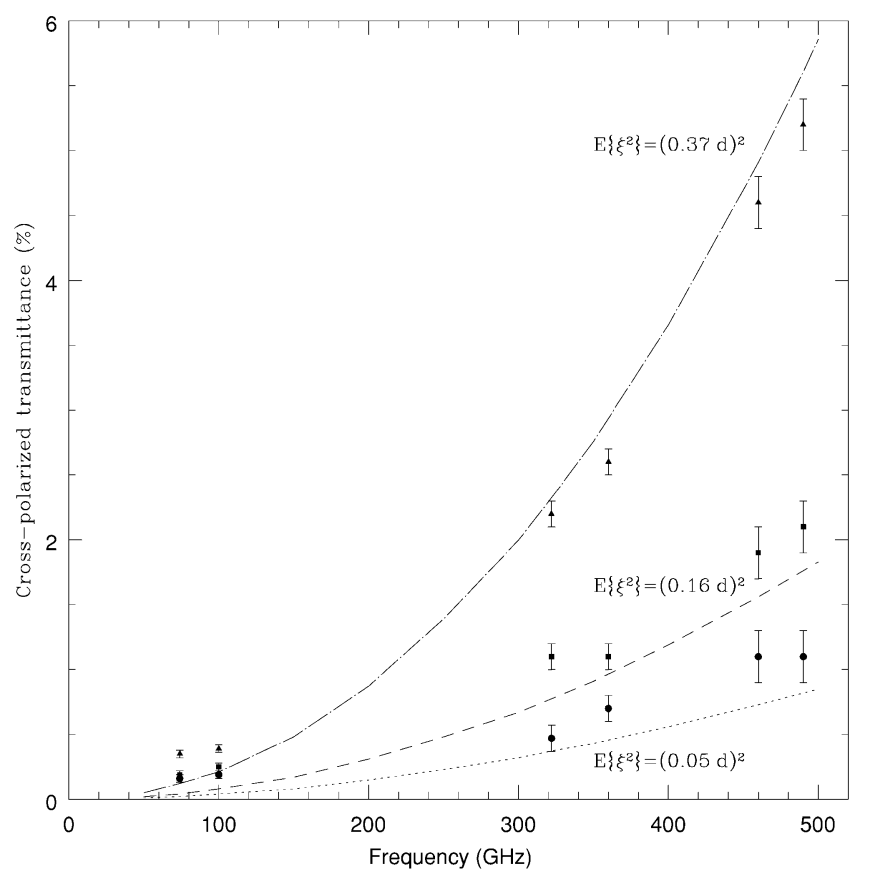

FIG. 3.-Curves of predicted values for the cross-polarized transmittance plotted against experimental data from Shapiro \& Bloemhof (1990) and E. E. Bloemhof (1998, private communication). The three grids have a random error $(1 \sigma)$ in wire positioning of $5 \%, 16 \%$, and $37 \%$ with mean distance between wires of $103 \mu \mathrm{m}, 109 \mu \mathrm{m}$, and $114 \mu \mathrm{m}$, respectively; they all have a wire radius of $12.5 \mu \mathrm{m}$

If the same approach is used to calculate the effect of such random errors on the value of the azimuthal surface current density $K_{n}^{\theta}$, one finds that it remains unaffected:

$$
\overline{K^{\theta}} \simeq K^{\theta}
$$

with $K^{\theta}$ given by equation (24).

From this we could then proceed and calculate the expected value of the reflection and transmission coefficients by evaluating equation (42) (and the corresponding equations for the $y$ and $z$ directions) in the far field; when this is accomplished we find that the coefficients have exactly the same form as shown in equations (30)-(35) (or eqs. [36]-[41]). We then merely have to replace $K^{x}$ and $K^{\theta}$ by $\overline{K^{x}}$ and $\overline{K^{\theta}}$, respectively.

\subsubsection{Random Errors in the Wire Radius (Wire to Wire)}

Another type of error that can be analyzed is one concerning the random variation in the size of the wire radius, which we will denote by the letter $\eta$. More explicitly, we are considering differences between wires and not variations along a single wire; we assume the diameter of a wire to be constant but somewhat uncertain in its value. This is the kind of phenomenon that could occur if the wires were stretched with slightly different tensions when installed or perhaps also in cases where the wires have a finite ellipticity and are rotated between rows.
We can proceed in the same manner as we did in the last section for the analysis of the boundary conditions and the fields away from the grid. When this is done we get

$$
\begin{aligned}
& \overline{K^{x}} \simeq K^{x}\left[1-\frac{E\left\{\eta^{2}\right\}}{\sum_{n=-\infty}^{\infty} G_{n}(0)}\left\{\frac{1}{2} \frac{\partial^{2} G_{0}}{\partial \eta_{0}^{2}}-\frac{\left(1-\alpha^{2}\right)}{\Delta_{x}}\left[\frac{\partial G_{0}}{\partial \eta_{0}}\right]^{2}\right\}_{\eta_{0}=0}\right], \\
& \overline{K^{\theta}} \simeq K^{\theta}\left[1-\frac{E\left\{\eta^{2}\right\}}{Q_{0}(0)}\left\{\frac{1}{2} \frac{\partial^{2} Q_{0}}{\partial \eta_{0}^{2}}+\frac{1}{a} \frac{\partial Q_{0}}{\partial \eta_{0}}-\frac{\sqrt{1-\alpha^{2}}}{\Delta_{\theta}}\left[\frac{\partial Q_{0}}{\partial \eta_{0}}\right]^{2}\right\}_{\eta_{0}=0}\right],
\end{aligned}
$$

where $\eta_{n}$ is the random error in the size of the radius of wire $n, G_{n}$ is given by equation (43) (with $\xi=0$ and $a$ replaced by $a+\eta), \Delta_{x}$ and $\Delta_{\theta}$ by equations (26) and (28), respectively, and $Q_{0}\left(\eta_{0}\right)=H_{1}^{(2)}\left[k^{\prime}\left(a_{0}+\eta_{0}\right)\right]$. Again the expected value of the different coefficients can be obtained by replacing the current components $K^{x}$ and $K^{\theta}$ by $\overline{K^{x}}$ and $\overline{K^{\theta}}$ in equations (30)-(35) (or eqs. [36]-[41]). It will also be noted that the errors contain a perturbation term that is proportional to their common variance.

\subsubsection{Predictions and Comparison with Experiments}

Now that we have derived the equations for the reflection and transmission coefficients, it would be interesting to compare the predictions that our model makes with experimental data. Although we have independently treated the two types of errors, it is nevertheless obvious that within the limit of precision of our analysis (small errors) that they can both be simultaneously added in the expressions for the reflection and transmission coefficients. Doing so would in principle allow us to compare theory and experiments as actual grids are liable to exhibit both kinds of defects. This also suggests though that it might be impossible to separate the effects of both errors in measurements. It turns out, however, that the perturbations caused by the errors in the size of the wire are predicted by our model to be smaller than those caused by the errors of the other type (for equivalent error amplitudes), and we neglect them in the following comparison of theory and measured grid properties.

Shapiro \& Bloemhof (1990) have published measurements of the unwanted cross-polarized transmittance through three grids on which they had purposely introduced random errors in the wire positioning. They quoted the errors in term of the random variation in the distance between wires (pitch) with amplitudes of $7 \%, 23 \%$, and $52 \%$ of the mean wire separation (aimed at $108 \mu \mathrm{m}$ with a wire radius of $12.5 \mu \mathrm{m}$ ). We must divide these values by a factor of $\sqrt{2}$ in order to relate them to our errors $\xi_{n}$ since we have defined these as pertaining to the absolute position of the wires. Figure 3 shows a comparison of our model's predictions with their measurements for cases where the incoming field is at normal incidence to the grid and polarized parallel to the wire orientation. Although the agreement is not perfect, the outcome is very satisfactory as the 
theoretical curves exhibit the right behavior with frequency and error amplitude.

\subsection{The Scattering Matrix and the Impedance Model}

\subsubsection{The Scattering Matrix and the Principal Axes of a Grid}

The relationship between the reflection and transmission coefficients in equations (36)-(41) is reminiscent of what is often encountered in microwave engineering in the analysis of systems that can be accurately dealt with using a lumped-elements model. With this in mind, it is tempting to consider any problem involving a polarizing grid by treating the different components as lumped and interconnected through a transmission line of characteristic impedance $Z_{0}$ (Lamb 1997). We can then go ahead and model the grid as a four-port device since the reflection and transmission coefficients given by the aforementioned set of equations provide us with the scattering parameters at each port.

In this context, it is more convenient to work with a single coordinate system $(u, v, w)$ (see Figs. 1 and 2 ) for both the incident/transmitted and reflected plane waves since we can assume that their propagation is done along the same transmission line (it is however understood that, in reality, away from normal incidence the transmitted and reflected waves travel along different axes). We therefore assume that the incident/transmitted fields travel along the $w$-axis (with the $u$-axis vertical and the $v$-axis horizontal) and the reflected fields along the negative $w$-axis as seen from a given side of the grid.

Since there are two possible independent states of polarization (with the field aligned along the $u$ - or $v$-axes), where the waves can travel either toward or away from the grid, we need two ports on each side of the grid. So for example, if the incident wave on a given port has an electric field polarized along a given axis we can define four scattering parameters: one for the reflected signal at the input port and three for the transmissions to the other ports. The same thing can be done for every port, leading to a total of 16 scattering parameters.

In what follows, a scattering parameter $s_{m n}$ is defined with the three ports $m \neq n$ terminated with the line characteristic impedance $Z_{0}$. Also, each port $n$ has two signals: an incoming signal $E_{n}^{+}$and an outgoing signal $E_{n}^{-} ; n=1,2(3,4$ on the other side of the grid) refer to polarization along the $u$ - and $v$-axes, respectively. The scattering matrix relates the different signals as follows:

$$
\left[\begin{array}{l}
E_{1}^{-} \\
E_{2}^{-} \\
E_{3}^{-} \\
E_{4}^{-}
\end{array}\right]=\left[\begin{array}{llll}
s_{11} & s_{12} & s_{13} & s_{14} \\
s_{21} & s_{22} & s_{23} & s_{24} \\
s_{31} & s_{32} & s_{33} & s_{34} \\
s_{41} & s_{42} & s_{43} & s_{44}
\end{array}\right]\left[\begin{array}{l}
E_{1}^{+} \\
E_{2}^{+} \\
E_{3}^{+} \\
E_{4}^{+}
\end{array}\right] .
$$

The elements of the matrix can be directly evaluated from equations (36)-(41) and shown to be

$$
S=\left[\begin{array}{llll}
R^{u u} & R^{u v} & T^{u u} & T^{u v} \\
R^{u v} & R^{v v} & T^{u v} & T^{v} \\
T^{u} & T^{v} & R^{u u} & R^{u v} \\
T^{v v} & T^{v v} & R^{u v} & R^{v v}
\end{array}\right]
$$

with

$$
\begin{aligned}
R^{u u} & =-\frac{\lambda}{\pi d} \frac{1}{\gamma\left(1-\gamma^{2}\right)}\left[\beta^{2} \frac{N_{x}}{\Delta_{x}}-\alpha^{2} \gamma^{2} \frac{k a}{2} \frac{N_{\theta}}{\Delta_{\theta}}\right], \\
R^{v v} & =-\frac{\lambda}{\pi d} \frac{1}{\gamma\left(1-\gamma^{2}\right)}\left[\alpha^{2} \gamma^{2} \frac{N_{x}}{\Delta_{x}}-\beta^{2} \frac{k a}{2} \frac{N_{\theta}}{\Delta_{\theta}}\right], \\
R^{u v} & =-\frac{\lambda}{\pi d} \frac{\alpha \beta}{\left(1-\gamma^{2}\right)}\left[\frac{N_{x}}{\Delta_{x}}+\frac{k a}{2} \frac{N_{\theta}}{\Delta_{\theta}}\right], \\
T^{u u} & =1-\frac{\lambda}{\pi d} \frac{1}{\gamma\left(1-\gamma^{2}\right)}\left[\beta^{2} \frac{N_{x}}{\Delta_{x}}+\alpha^{2} \gamma^{2} \frac{k a}{2} \frac{N_{\theta}}{\Delta_{\theta}}\right], \\
T^{v v} & =1-\frac{\lambda}{\pi d} \frac{1}{\gamma\left(1-\gamma^{2}\right)}\left[\alpha^{2} \gamma^{2} \frac{N_{x}}{\Delta_{x}}+\beta^{2} \frac{k a}{2} \frac{N_{\theta}}{\Delta_{\theta}}\right], \\
T^{u v} & =-\frac{\lambda}{\pi d} \frac{\alpha \beta}{\left(1-\gamma^{2}\right)}\left[\frac{N_{x}}{\Delta_{x}}-\frac{k a}{2} \frac{N_{\theta}}{\Delta_{\theta}}\right],
\end{aligned}
$$

where $N_{x}, \Delta_{x}, N_{\theta}$, and $\Delta_{\theta}$ are given by equations (25), (26), (27), and (28), respectively.

We can go one step further and render things considerably simpler if we make a change of coordinates and use the following as eigenvectors instead of $\boldsymbol{e}_{\boldsymbol{u}}$ and $\boldsymbol{e}_{\boldsymbol{v}}$ :

$$
\boldsymbol{p}_{1}=\frac{\beta \boldsymbol{e}_{u}+\alpha \gamma \boldsymbol{e}_{v}}{\sqrt{\beta^{2}+\alpha^{2} \gamma^{2}}}, \quad \boldsymbol{p}_{2}=\frac{-\alpha \gamma \boldsymbol{e}_{u}+\beta \boldsymbol{e}_{v}}{\sqrt{\beta^{2}+\alpha^{2} \gamma^{2}}}
$$

From now on we will refer to these as the principal axes of the grid (for reasons that will soon become apparent). A close examination of the first of these two equations shows that $\boldsymbol{p}_{1}$ is parallel to the projection of the direction of the wires in the plane of the incident field. The matrix $S$ then takes a simpler form (we also interchange the second row with the third and 
the second column with the third):

$$
S=\left[\begin{array}{cccc}
R_{\|} & T_{\|} & 0 & 0 \\
T_{\|} & R_{\|} & 0 & 0 \\
0 & 0 & R_{\perp} & T_{\perp} \\
0 & 0 & T_{\perp} & R_{\perp}
\end{array}\right],
$$

where

$$
\begin{aligned}
R_{\|} & =-\frac{\lambda}{\pi d} \frac{\left(1-\alpha^{2}\right)}{\gamma} \frac{N_{x}}{\Delta_{x}}, \\
R_{\perp} & =\frac{\left(1-\alpha^{2}\right)}{\gamma} \frac{a}{d} \frac{N_{\theta}}{\Delta_{\theta}}, \\
T_{\|} & =1+R_{\|}, \\
T_{\perp} & =1-R_{\perp} .
\end{aligned}
$$

We then have a further simplification in the modeling of the grid; evidently equations (57)-(60) represent the reflection and transmission coefficients along the two principal axes.

This last representation has the advantage of simplifying calculations since it allows us to decompose any incident field into two noninteracting components, one along each one of the principal axes. That is, a field polarized along one of the principal axes scatters only in this same polarization state (as can be deduced from the block-diagonal form of eq. [56]). It is also interesting to note that even though we have defined the principal axes within the framework of our approximation of the grid $\left(k^{\prime} a \ll 1\right.$ and $\left.a \ll d\right)$, the result obtained here still holds in the general case (see Appendix A for a proof). This implies that for the case where one wishes to use a different approach to solve (numerically or otherwise) the scattering off a grid of arbitrary characteristics, it will always be possible to split the incoming field along the principal axes, thereby avoiding crosspolarization terms and greatly simplifying the solution.

\subsubsection{The Impedance Model}

It seems reasonable to think that a grid could also be modeled with another representation where the scattering matrix is replaced by an impedance matrix that contains the same number of elements since, as before, the grid is still treated as a fourport device. In this case, however, the matrix relates the total voltages (electric fields) and currents (magnetic fields) between each and every port (Collin 1992, pp. 233-257). The scattering matrix formulation follows more naturally from our analysis and has the advantage of dealing with quantities (reflection and transmission coefficients) that are directly measurable, whereas impedances are not (at least at the wavelengths considered here). The impedance model has, however, received a great deal of attention in the literature and often seems to be the way in which polarizing grids are characterized (Wait 1954, 1955; Larsen 1962).

Taking advantage of the principal axes representation, it is possible to treat each two-dimensional block of the scattering matrix (eq. [56]) separately. It can be shown that the impedance matrix $\boldsymbol{Z}_{b}$ corresponding to a given block $\boldsymbol{S}_{b}$ can be expressed as

$$
\boldsymbol{Z}_{b}=Z_{0} \cdot\left(\boldsymbol{I} \pm \boldsymbol{S}_{b}\right) \cdot\left(\boldsymbol{I} \mp \boldsymbol{S}_{b}\right)^{-1},
$$

where $\boldsymbol{I}$ is the unit matrix and the upper and lower signs correspond, respectively, to the upper left and lower right blocks of the scattering matrix (eq. [56]). Applying this last equation to equation (56) we get

$$
\boldsymbol{Z}=\left[\begin{array}{cccc}
Z_{p} & Z_{p} & 0 & 0 \\
Z_{p} & Z_{p} & 0 & 0 \\
0 & 0 & Z_{n} & -Z_{n} \\
0 & 0 & -Z_{n} & Z_{n}
\end{array}\right]
$$

with

$$
Z_{p}=-\frac{Z_{0}}{2} \frac{1+R_{\|}}{R_{\|}}, \quad Z_{n}=\frac{Z_{0}}{2} \frac{1-R_{\perp}}{R_{\perp}} .
$$

It follows quite naturally from equations (57)-(60) that we could have defined two impedances $Z_{\|}$and $Z_{\perp}$ :

$$
Z_{\|}=Z_{0} \frac{1+R_{\|}}{1-R_{\|}}, \quad Z_{\perp}=Z_{0} \frac{1-R_{\perp}}{1+R_{\perp}}
$$

One can easily verify that $Z_{\|}$and $Z_{\perp}$ are, respectively, equal to $Z_{p}$ and $Z_{n}$ placed in parallel to the characteristic impedance $Z_{0}$. We therefore see that the impedance matrix gives the "actual" impedance of the grid along each of the principal axes, whereas the scattering matrix includes, as should be expected, the contribution of the loads of characteristic impedance $Z_{0}$, which is assumed to be connected to the appropriate ports when defining its parameters.

\subsection{Approximations and Selection of a Grid}

We will now study more closely our simpler equations (57) and (58) for the reflection coefficients $R_{\|}$and $R_{\perp}$ and try to find relations that will allow us to find a set of optimum parameters for the selection of a grid. But before we do so, it will be to our advantage to approximate the expressions for $\Delta_{x}$ and $\Delta_{\theta}$ (eqs. [26] and [28]).

So if we limit ourselves to situations where $d \ll \lambda, a \ll \lambda$, and $Z_{s} \ll Z_{0}$ (good conducting wires) and use the proper expansion for Hankel's functions and series of Hankel's functions 
applicable in such cases (small arguments), we find

$$
\begin{aligned}
& \Delta_{x} \simeq\left(1-\alpha^{2}\right)\left(\left\{\frac{\lambda}{\pi \gamma d}-\left(\frac{k^{\prime} a}{2}\right)^{2}+\frac{1}{\sqrt{\left(1-\alpha^{2}\right) \pi Z_{0} \sigma \lambda}}\left(\frac{2}{k^{\prime} a}\right)\right\}\right. \\
& +j \frac{2}{\pi}\left\{\ln \left(\frac{d}{2 \pi a}\right)+\frac{\pi^{2}}{6}\left(\frac{d \gamma}{\lambda}\right)^{2}\right. \\
& -\left(\frac{k^{\prime} a}{2}\right)^{2}\left[1-\Psi-\ln \left(\frac{k^{\prime} a}{2}\right)\right] \\
& \left.\left.+\sqrt{\frac{\pi}{4\left(1-\alpha^{2}\right) Z_{0} \sigma \lambda}}\left(\frac{2}{k^{\prime} a}\right)\right\}\right), \\
& \Delta_{\theta} \simeq-\left(1-\alpha^{2}\right) \frac{Z_{s}}{Z_{0}} \frac{2}{\pi} \ln \left(\frac{d}{2 \pi a}\right)+j\left[\frac{\lambda}{\pi^{2} a}+\left(1-\alpha^{2}\right) \frac{Z_{s}}{Z_{0}} \frac{\lambda}{\pi \gamma d}\right],
\end{aligned}
$$

where $\Psi \simeq 0.577215$ is Euler's constant. In equation (63) we kept things to the lowest order possible and did not expand $Z_{s}$; the same is not true for equation (62) for reasons that we shall encounter shortly.

We turn now to the problem of selecting the right parameters for a grid. If we decompose a given incident field into two components along the principal axes $\boldsymbol{p}_{1}$ and $\boldsymbol{p}_{2}$ (see $\S 3.3 .1$ ), a perfect grid would completely reflect the first of these and transmit the second $\left(R_{\|}=-1\right.$ and $\left.T_{\perp}=1\right)$. As we will soon see, the coefficient of reflection $R_{\perp}$ is proportional to $a^{2} / d \lambda$ when $Z_{s} \rightarrow 0$ and is therefore a very small quantity for the cases considered here; thus we will not worry about it anymore (i.e., $T_{\perp}$ is nearly equal to unity). The condition of total reflection will dictate our choice for the parameters of the grid. A close study of equation (57) tells us that in order to achieve perfect reflection we must simultaneously satisfy the following relations for the real and imaginary parts of $\Delta_{x}$ (for what follows we assume $N_{x} \simeq 1$; see eq. [25]):

$$
\operatorname{Re}\left\{\Delta_{x}\right\}=\frac{\lambda}{\pi d} \frac{\left(1-\alpha^{2}\right)}{\gamma}, \quad \operatorname{Im}\left\{\Delta_{x}\right\}=0
$$

Solving for these we then get

$$
\begin{aligned}
& a \simeq\left[\frac{\lambda^{5}}{\left(1-\alpha^{2}\right)^{4} \pi^{7} \sigma Z_{0}}\right]^{1 / 6}, \\
& d \simeq 2 \pi a .
\end{aligned}
$$

Had we kept equation (62) to the lowest order, we would have been unable to specify an optimum value for the wire radius but only the relation that binds $d$ to $a$. It is also of interest to note that for a given wavelength, the finite size of the wire radius is, to this level of approximation, dictated by the conductivity $\sigma$; if we let $\sigma \rightarrow \infty$ then there is no restriction on the smallness of the radius.

In a quantitative example to demonstrate the values that can be expected for $a$ and $d$, assume that we are working at normal incidence at a wavelength of $1 \mathrm{~mm}$ with a grid made of copper $\left(\sigma=5.8 \times 10^{7} \Omega^{-1} \mathrm{~m}^{-1}\right)$. Using these, we obtain $a \simeq 11 \mu \mathrm{m}$ and $d \simeq 70 \mu \mathrm{m}$.

At this point it is appropriate to discuss the implications of the two assumptions we made at the beginning concerning the wire radius and spacing, namely, that $k^{\prime} a \ll 1$ and $a \ll d$. It is important to make sure that a given choice of grid parameters are well within the boundaries of applicability of our model. As a means of determining these boundaries, we simulated the response of grids (and assemblies of grids; see $\S 4$ ) for different combinations of wire radius and spacing and made sure that the results obtained were reliable (for example, it is obviously imperative that the magnitude of the reflection and transmission coefficients never exceed unity). As it turns out, there is a fairly strong restriction linking the size of the wires and the wavelength, but if one makes sure that $\lambda>40 a$, then one seems to be well within safe modeling conditions. The value of $a$ cannot be too small either. However, since for a good conductor (again let us use copper) the skin depth at $1 \mathrm{~mm}$ is on the order of $0.1 \mu \mathrm{m}$, our assumption of the existence of an idealized surface current is more than adequate. It seems that the second restriction concerning the spacing of the wires is not as binding as the first one. It is clear that $d>2 a$, for if not the wires would be touching, but it appears that everything is fine for $d>4 a$. Our proposed optimized values for the grid are therefore justified.

It is also appropriate to point out that using equations (62) and (63) for $\Delta_{x}$ and $\Delta_{\theta}$ (with or without the optimal values for $a$ and $d$ given by eqs. [64] and [65]) along with equations (57)-(60) for the reflection and transmission coefficients along the principal axes renders the task of calculating the response of a grid a rather simple one. It becomes unnecessary to confront the more intimidating representations derived earlier in $\S 3.1$ (compare with eqs. [23]-[35]). For example, to the lowest order, we get for the reflection coefficients:

$$
\begin{aligned}
& R_{\|} \simeq \frac{-1}{1+j[(2 \gamma d) / \lambda] \ln [d /(2 \pi a)]}, \\
& R_{\perp} \simeq-j \frac{\left(1-\alpha^{2}\right)}{\gamma} \frac{\pi^{2} a^{2}}{\lambda d},
\end{aligned}
$$

which are in agreement with known results (Larsen 1962) (more precisely, for the case of normal incidence discussed in Larsen 1962, eq. [66] reduces to the result presented there, whereas eq. [67] differs by a factor of 2 or 3 depending on which approximation it is compared to). 


\section{THE REFLECTING POLARIZER}

\subsection{Analysis}

From the solution of the polarizing grid it is a somewhat natural extension to consider the more complicated problem of the reflecting polarizer. A reflecting polarizer consists of an assembly where a polarizing grid, like the one studied in the last section, is followed by a mirror paralleling it at some distance $z_{0}$ behind (effectively placing the mirror at $z=0$ ).

It is appropriate in this case to use the method of images to solve this problem (Wait 1954). We then assume that images of both the incident and scattered fields are emanating from the other side of the mirror. This is equivalent to saying that the image world is made of a grid positioned at $z=-z_{0}$ with an image incident field impinging on it. Assuming that the mirror is made of a material of good conductivity, one can write for the image incident field $\boldsymbol{E}_{i}^{\prime}(\boldsymbol{r})$ :

$$
\boldsymbol{E}_{i}^{\prime}(\boldsymbol{r})=E_{0}\left(\alpha_{m}^{\prime} \boldsymbol{e}_{x}+\beta_{m}^{\prime} \boldsymbol{e}_{y}-\gamma_{m}^{\prime} \boldsymbol{e}_{z}\right) \exp [-j k(\alpha x+\beta y-\gamma z)]
$$

with

$$
\begin{aligned}
& \alpha_{m}^{\prime}=\frac{1}{\left(1-\gamma^{2}\right)}\left[\alpha^{\prime}\left(\alpha^{2} R_{\mathrm{TM}}+\beta^{2} R_{\mathrm{TE}}\right)+\alpha \beta \beta^{\prime}\left(R_{\mathrm{TM}}-R_{\mathrm{TE}}\right)\right], \\
& \beta_{m}^{\prime}=\frac{1}{\left(1-\gamma^{2}\right)}\left[\beta^{\prime}\left(\alpha^{2} R_{\mathrm{TE}}+\beta^{2} R_{\mathrm{TM}}\right)+\alpha \beta \alpha^{\prime}\left(R_{\mathrm{TM}}-R_{\mathrm{TE}}\right)\right], \\
& \gamma_{m}^{\prime}=\gamma^{\prime} R_{\mathrm{TM}},
\end{aligned}
$$

where $R_{\mathrm{TE}}$ and $R_{\mathrm{TM}}$ are the reflection coefficients of the mirror, with a dependency on the angle of incidence, for transverse electric and transverse magnetic modes of incoming radiation, respectively (Fowles 1975). It is important to note that these tranverse modes of radiation are not the same as those introduced in $\S 2$; they are defined here in relation to the plane which is parallel the normal vector out of the surface of the mirror $\left(-\boldsymbol{e}_{z}\right)$ and the wavevector $\boldsymbol{k}$.

One can go through calculations similar to those carried out in $\S 3.1$ and find the following relations between the components of the total surface current densities of the "real" and image grids:

$$
\begin{aligned}
& K^{\prime x}=\frac{\alpha_{m}^{\prime}}{\alpha^{\prime}} K^{x}, \\
& K^{\prime \theta}=-\frac{\gamma_{m}^{\prime} \beta-\beta_{m}^{\prime} \gamma}{\gamma^{\prime} \beta-\beta^{\prime} \gamma} K^{\theta},
\end{aligned}
$$

where $\boldsymbol{K}^{\prime}$ stands for the surface current of the image grid.

From these and by matching the boundary conditions at the grid, it is straightforward, but tedious, to solve for the problem.
We give here the final results:

$$
\begin{aligned}
K^{x}= & \frac{E_{0}}{F} \frac{2 j \alpha^{\prime} \operatorname{sn}(k \gamma h) N_{x}}{\left(1-\alpha^{2}\right) \Delta S_{1}-j\left(Z_{s} / Z_{0}\right) \sqrt{1-\alpha^{2}} \Delta S_{2}} \\
& \times \exp (-j k \gamma h), \\
K^{\theta}= & \frac{E_{0}}{F} \frac{-2 j\left(\gamma^{\prime} \beta-\beta^{\prime} \gamma\right) \operatorname{cs}(k \gamma h) N_{\theta}}{\sqrt{1-\alpha^{2}} \Sigma S_{2}+j\left(Z_{s} / Z_{0}\right)\left(1-\alpha^{2}\right) \Sigma S_{1}} \\
& \times \exp (-j k \gamma h), \\
R^{x}= & \alpha_{m}^{\prime}-\frac{F}{E_{0}} \frac{2 \lambda}{\pi d} \frac{\left(1-\alpha^{2}\right)}{\gamma} j \operatorname{sn}(k \gamma h) K^{x} \exp (j k \gamma h), \\
R^{y}= & \beta_{m}^{\prime}+\frac{F}{E_{0}} j \frac{2 \lambda}{\pi d}\left[\frac{\alpha \beta}{\gamma} \operatorname{sn}(k \gamma h) K^{x}-\frac{k a}{2} \operatorname{cs}(k \gamma h) K^{\theta}\right] \\
& \times \exp (j k \gamma h), \\
R^{z}= & -\gamma_{m}^{\prime}-\frac{F}{E_{0}} j \frac{2 \lambda}{\pi d}\left[\alpha \operatorname{sn}(k \gamma h) K^{x}+\frac{\beta}{\gamma} \frac{k a}{2} \operatorname{cs}(k \gamma h) K^{\theta}\right] \\
& \times \exp (j k \gamma h),
\end{aligned}
$$

where

$$
\begin{aligned}
\operatorname{sn}(x)= & \frac{1}{2 j}\left[\exp (j x)-r_{x} \exp (-j x)\right], \\
\operatorname{cs}(x)= & \frac{1}{2}\left[\exp (j x)+r_{\theta} \exp (-j x)\right], \\
\Delta S_{1}= & H_{0}^{(2)}\left(k^{\prime} a\right)-r_{x} H_{0}^{(2)}\left(k^{\prime} 2 h\right) \\
& +2 \sum_{n=1}^{\infty}\left\{H_{0}^{(2)}\left(k^{\prime} n d\right)-r_{x} H_{0}^{(2)}\left[k^{\prime} \sqrt{(n d)^{2}+4 h^{2}}\right]\right\} \\
& \times \cos (k \beta n d), \\
\Delta S_{2}= & H_{1}^{(2)}\left(k^{\prime} a\right)-r_{x} H_{1}^{(2)}\left(k^{\prime} 2 h\right), \\
\Sigma S_{1}= & H_{0}^{(2)}\left(k^{\prime} a\right)+r_{\theta} H_{0}^{(2)}\left(k^{\prime} 2 h\right) \\
& +2 \sum_{n=1}^{\infty}\left\{H_{0}^{(2)}\left(k^{\prime} n d\right)+r_{\theta} H_{0}^{(2)}\left[k^{\prime} \sqrt{(n d)^{2}+4 h^{2}}\right]\right\} \\
& \times \cos (k \beta n d), \\
\Sigma S_{2}= & H_{1}^{(2)}\left(k^{\prime} a\right)+r_{\theta} H_{1}^{(2)}\left(k^{\prime} 2 h\right),
\end{aligned}
$$

and

$$
r_{x}=-\frac{\alpha_{m}^{\prime}}{\alpha^{\prime}}, \quad r_{\theta}=-\frac{\gamma_{m}^{\prime} \beta-\beta_{m}^{\prime} \gamma}{\gamma^{\prime} \beta-\beta^{\prime} \gamma} .
$$

We have also replaced $-z_{0}$ by $h(h>0)$ so that the distance between the mirror and the grid is expressed by a positive quantity. This set of equations along with equations (68)-(70) 
give us the solution of the reflecting polarizer problem for cases where $k^{\prime} a \ll 1$ and $a \ll d$.

As was the case for the polarizing grid, if we transform those coefficients to the laboratory frame of coordinates $\left(u^{\prime}, v^{\prime}, w^{\prime}\right)$ (see Fig. 2) we obtain

$$
\begin{aligned}
R^{u^{\prime}}= & \alpha_{m}^{\prime \prime}-\frac{F}{E_{0}} \frac{2 \lambda}{\pi d} \frac{j \exp (j k \gamma h)}{\gamma \sqrt{1-\gamma^{2}}} \\
& \times\left[\beta \operatorname{sn}(k \gamma h) K^{x}-\alpha \gamma \frac{k a}{2} \operatorname{cs}(k \gamma h) K^{\theta}\right], \\
R^{v^{\prime}}= & \beta_{m}^{\prime \prime}-\frac{F}{E_{0}} \frac{2 \lambda}{\pi d} \frac{j \exp (j k \gamma h)}{\gamma \sqrt{1-\gamma^{2}}} \\
& \times\left[\alpha \gamma \operatorname{sn}(k \gamma h) K^{x}+\beta \frac{k a}{2} \operatorname{cs}(k \gamma h) K^{\theta}\right], \\
R^{w^{\prime}}= & 0
\end{aligned}
$$

with $\alpha_{m}^{\prime \prime}$ and $\beta_{m}^{\prime \prime}$ given by

$$
\begin{aligned}
\alpha_{m}^{\prime \prime}= & \frac{1}{\left(1-\gamma^{2}\right)}\left[\alpha^{\prime \prime}\left(\alpha^{2} R_{\mathrm{TM}}+\beta^{2} R_{\mathrm{TE}}\right)\right. \\
& \left.+\alpha \beta \beta^{\prime \prime}\left(R_{\mathrm{TM}}-R_{\mathrm{TE}}\right)\right] \\
\beta_{m}^{\prime \prime}= & \frac{1}{\left(1-\gamma^{2}\right)}\left[\beta^{\prime \prime}\left(\alpha^{2} R_{\mathrm{TE}}+\beta^{2} R_{\mathrm{TM}}\right)\right. \\
& \left.+\alpha \beta \alpha^{\prime \prime}\left(R_{\mathrm{TM}}-R_{\mathrm{TE}}\right)\right]
\end{aligned}
$$

and finally $\alpha^{\prime \prime}$ and $\beta^{\prime \prime}$ are related to the incident field by

$$
\boldsymbol{E}_{i}(\boldsymbol{r})=E_{0}\left(\alpha^{\prime \prime} \boldsymbol{e}_{u}+\beta^{\prime \prime} \boldsymbol{e}_{v}\right) \exp (-j k w)
$$

\subsection{Solution Using the Scattering Matrix}

The scattering matrix representation of the polarizing grid gives us the advantage of rendering possible the solution of problems that would be otherwise extremely difficult, if not impossible, to solve using Maxwell's equations. For example, a solution of the reflecting polarizer problem is straightforward if we "connect" the mirror to ports 3 and 4 of the grid at a distance $h$ behind. Using the definitions introduced in the discussion leading to equation (48) we have

$$
\begin{aligned}
& E_{3}^{+}=E_{3}^{-} R_{\mathrm{TE}} \exp (-j k \gamma 2 h), \\
& E_{4}^{+}=E_{4}^{-} R_{\mathrm{TM}} \exp (-j k \gamma 2 h),
\end{aligned}
$$

where $R_{\mathrm{TE}}$ and $R_{\mathrm{TM}}$ are as defined in the previous section.

We can then solve for $E_{1}^{-}$and $E_{2}^{-}$and find results that are in agreement with those obtained in the previous section.

\subsection{Experimental Results}

Reflecting polarizers like those studied here were tested at the Owens Valley Radio Observatory (OVRO) for polarimetry in the wavelength ranges of $1.3 \mathrm{~mm}$ as well as successfully used for polarimetry observations at $3 \mathrm{~mm}$ (Akeson 1997; Akeson et al. 1996). They are composed of an aluminum mirror and a grid of gold-plated tungsten wires of $25 \mu \mathrm{m}$ diameter and spaced at an interval of $125 \mu \mathrm{m}$. The inside diameter of the grid is roughly $16 \mathrm{~cm}$, some 25 times larger than the incident Gaussian beam at $1.3 \mathrm{~mm}$ (Akeson 1997).

In this section, we will compare data obtained in the calibration of these polarizers at $1.3 \mathrm{~mm}$ with the model calculated earlier. In the experimental setup, the incident beam is composed of radiation emanating from a hot load (absorber at room temperature) polarized along the vertical axis and a cold load (absorber in liquid nitrogen) along the horizontal axis. The beam is incident on the polarizers at an angle of $\chi_{i}=34^{\circ}$ with the grid rotated by $\varphi_{g}= \pm 50^{\circ} .3$ relative to the vertical, all in the coordinate system of the laboratory (coordinates $(u, v, w)$ of Fig. 1). These values can be inserted in the appropriate equations of our earlier analysis and used to test our model against the experimental data.

The calibration consists in using our model to map out the actual distance between the grid and the mirror as the latter is moved with a micropositioner which is part of the assembly. When this is done, this distance can then be precisely adjusted to $\lambda / 8 \gamma$ in order to use the polarizer as a reflecting quarterwave plate for polarimetry measurements. The grid rotation angle $\varphi_{g}$ must also be calibrated so that it can be set to the proper value that will allow the transformation of incident linear polarization to circular polarization. (This condition is met for $\beta= \pm \alpha \gamma\left[\right.$ or $\left.\tan \left(\varphi_{g}\right)= \pm 1 / \cos \left(\chi_{i}\right)\right]$, as can be asserted from our earlier discussion of the principal axes of a grid in $\S 3.3$; this gives $\varphi_{g}= \pm 50^{\circ} 3$ as quoted above).

Figure 4 shows the results obtained from such measurements (of the reflected polarized intensity along the horizontal $v^{\prime}$-axis in the laboratory coordinate system) made on antenna 6 of the OVRO array at a frequency of $232.037 \mathrm{GHz}$ when the separation between the grid and the mirror is varied through a range of several hundreds of microns. Accompanying the data points is a least-squares fit of our model (solid curve) with no free parameters as far as the grid is concerned; only the hot and cold load levels and the offset in the mirror-grid separation were allowed to be fitted. The agreement is very good. The main shortcoming of the fit is at a backshort position of roughly $900 \mu \mathrm{m}$ where a resonance is evident from the data. The model also shows a resonance at the same position, but the fit is not perfect. This feature is caused by the small amount of unwanted transmission from the component of the incident electric field aligned with one of the principal axes $\left(\boldsymbol{p}_{1}\right)$ which gets trapped between the grid and the mirror.

Before we try to explain the differences in width and shape of the resonance, we would first like to show two ways by 


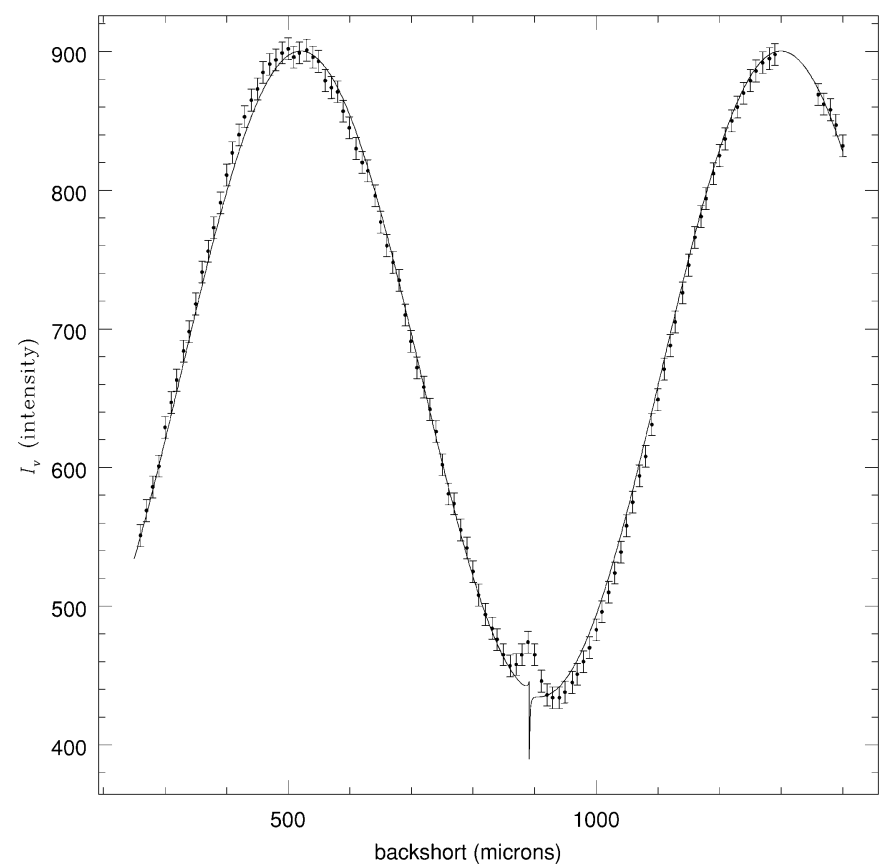

FIG. 4.-Calibration data from antenna 6 of the OVRO array. Data points are shown with an error bar and the solid curve is a least-squares fit from the model presented in this paper. The intensity is in arbitrary units.

which it can be suppressed (such a response from the polarizer is a nuisance when trying to calibrate it and should be avoided).

First, reducing the amount of unwanted transmission through the grid would certainly have a damping effect on the resonance. We have shown how to do just that in $\S 3.4$ when defining a set of optimum parameters for a grid, so using equations (64) and (65) we find for our application $a \simeq 24 \mu \mathrm{m}$ and $d \simeq 148 \mu \mathrm{m}$. Figure 5 shows a comparison of the simulated responses for the polarizer tested at OVRO and our optimized polarizer. As can be seen, any sign of the resonance has disappeared in the latter.

Another way of avoiding the resonance, while still using the same original grid with $a=12.5 \mu \mathrm{m}$ and $d=125 \mu \mathrm{m}$, is to replace the mirror with another grid (K. H. Young 1997, private communication) and rotate both of them in such a way that the projected orientation of their wires in the plane of the incident field is aligned with a principal axis. By this we mean that the two grids have their angles of rotation specified by $\tan \left(\varphi_{g}\right)=1 / \cos \left(\chi_{i}\right)$ (or $\varphi_{g}=50^{\circ} 3$ ) and $\tan \left(\varphi_{g}\right)=-1 / \cos \left(\chi_{i}\right)$ (or $\varphi_{g}=-50^{\circ} 3$ ), respectively. This would ensure that the unwanted transmitted field from the first grid would almost be entirely transmitted through the second grid, therefore eliminating the resonance.

Obviously, trying to solve for such a configuration using Maxwell's equations would be a formidable task. We can however use our scattering matrix model developed in $\S 3.3$. We then have to define two matrices, one for each grid, and solve the problem for cases where they are separated by a given

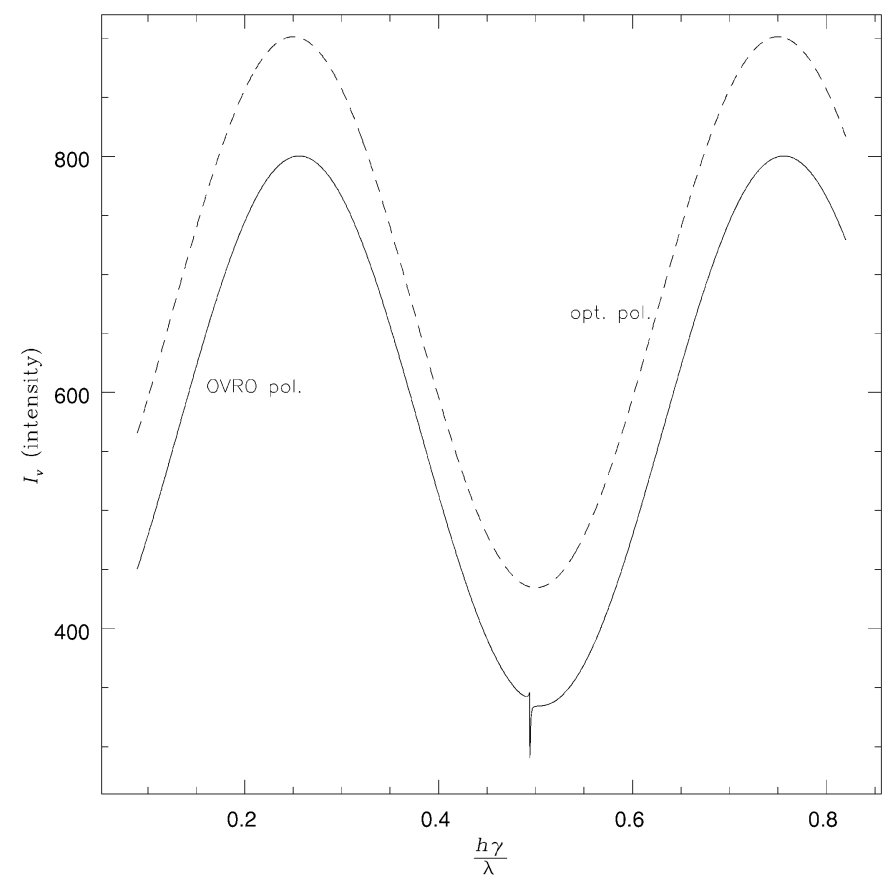

FIG. 5.-Comparison of the predicted results obtained for the polarizer tested at OVRO (solid curve) and our optimized polarizer (dashed curve). The resonance is not present on the optimized polarizer's response. The two curves are plotted with a small vertical offset between them. The intensity is in arbitrary units.

distance while terminating the last grid by the line characteristic impedance $Z_{0}\left(E_{3}^{+}=E_{4}^{+}=0\right)$. We simulated the response predicted for such an arrangement of grids and got results that are practically identical to those presented in Figure 5 for the optimum polarizer.

\section{GRIDS AND BEAMS OF RADIATION}

Until now we have restricted our analysis to cases where the dimensions of the grid (or the assembly) and the extent of the incident wave were assumed to be infinite. These simplifications were necessary in order to allow us to have a chance at a solution, as the reality of finite sizes introduces severe difficulties in the analysis. It would, however, seem reasonable to suppose that if the incoming excitation can be properly represented by a beam of radiation which is of a size a few times smaller that the actual dimensions of the assembly, the results obtained with our analysis should still be valid. Indeed, one could argue that the incident beam should induce currents only in the vicinity of the area where it impinges on the assembly. There should therefore be little to no difference in its response depending on whether it is infinite or not.

Although we believe this argument to be a reasonable one, we will show that the characteristics of the incoming radiation can be important in some cases. We will in fact argue that it can explain the discrepancies in the width and shape of the 


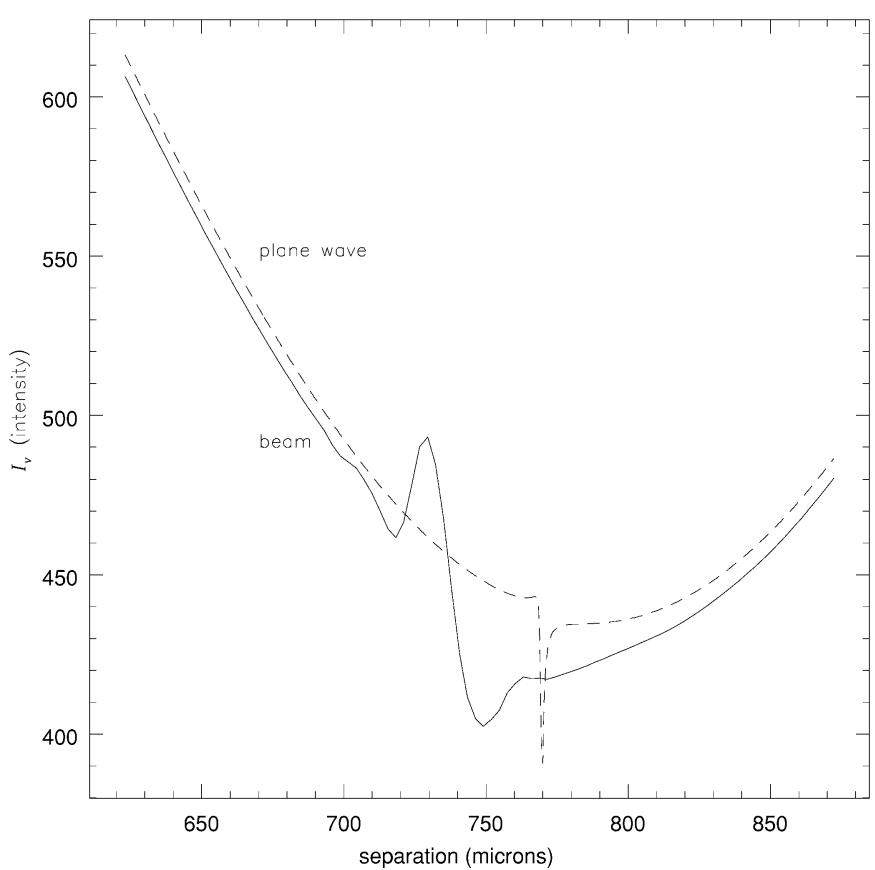

FIG. 6.-Simulation of the effect of a Gaussian beam $\left(W_{0}=3 \mathrm{~mm}\right)$ on the width and shape of the resonance exhibited by a reflecting polarizer as discussed in $\S 4.3$. The broken and solid curves show the results predicted for an incident plane wave and a Gaussian beam, respectively.

resonance observed in the response of the reflecting polarizer presented in $\S 4.3$ (see Fig. 4).

As a starting point, let us take note that we can always mathematically express a beam of radiation $E_{0}(\boldsymbol{r})$ as a summation of plane waves with different amplitude (and phase) and $\boldsymbol{k}$ vectors. For example, in the laboratory system of coordinates $(u, v, w)$, the electric field along the $u$-axis $E_{i}^{u}(\boldsymbol{r}, t)=\alpha^{\prime \prime} E_{0}(\boldsymbol{r}) \exp \left(j \omega_{0} t\right)$ can be expressed by (using its Fourier transform in $(\omega, \boldsymbol{k})$-space and assuming the beam to be monochromatic at $\omega_{0}=k c$ ):

$$
\begin{aligned}
E_{i}^{u}(\boldsymbol{r}, t)= & \frac{\alpha^{\prime \prime}}{(2 \pi)^{4}} \int_{-\infty}^{\infty} d \omega d^{3} k E_{0}(\boldsymbol{k}, \omega) \\
& \times \exp [-j(\boldsymbol{k} \cdot \boldsymbol{r}-\omega t)], \\
E_{0}(\boldsymbol{k}, \omega)= & 2 \pi \delta\left(\omega-\omega_{0}\right) \int_{-\infty}^{\infty} d^{3} r^{\prime} E_{0}\left(\boldsymbol{r}^{\prime}\right) \exp \left(j \boldsymbol{k} \cdot \boldsymbol{r}^{\prime}\right),
\end{aligned}
$$

where $\delta(x)$ is Dirac's delta distribution.

Let us now assume that the incident radiation can be satisfactorily modeled using a circular Gaussian beam with a beam waist $W_{0}$ and a Rayleigh range $z_{\mathrm{R}}=\pi W_{0}^{2} / \lambda$. We also know that the resonance will occur for a grid-mirror separation of $h \simeq \pi / k_{z}$ for each spectral component, where $k_{z}$ is the projection of the wavevector along the $z$-axis perpendicular to the reflecting polarizer (in the coordinates system of the grid of Fig. 1).
From this we can express the width of the resonance $\Delta h$ as a function of $k_{z}$ and $\Delta k_{z}$ the spectrum extent along the same axis:

$$
\Delta h \simeq \pi \frac{\Delta k_{z}}{k_{z}^{2}} .
$$

We need to find an expression for $\Delta k_{z}$ and this can be done as follows. Using the wave uncertainty relation, we can evaluate the spectrum extent in the laboratory system of axes as

$$
\Delta k_{u} \simeq W_{0}^{-1}, \quad \Delta k_{v} \simeq W_{0}^{-1}, \quad \Delta k_{w} \simeq z_{\mathrm{R}}^{-1} .
$$

Transforming these in the coordinate system of the grid and inserting the result in the expression for the width of the resonance, we find

$$
\Delta h \simeq \frac{\pi}{\left(k \gamma W_{0}\right)^{2}} \sqrt{W_{0}^{2}\left(1-\gamma^{2}\right)+\left(\frac{2 \gamma}{k}\right)^{2}} .
$$

When we use the corresponding values of the different parameters appearing in equation (83) for the case of the reflecting polarizer discussed in the last section $(\lambda=1.3 \mathrm{~mm}, \gamma=0.83$, and $W_{0}=3 \mathrm{~mm}$ ), we find $\Delta h \simeq 37 \mu \mathrm{m}$. If we take into account the finite bandwidth of the OVRO receivers $(1 \mathrm{GHz})$, a similar exercise shows that at most only a few microns need to be added to the previous estimate. Although these numbers represent only a rough calculation of what could be expected, they nevertheless tell us that there will be a significant broadening of the resonant feature.

We will not try to produce a perfect fit to the data obtained at OVRO, for this would require extensive modeling of our experimental setup and therefore bring us to a level of complexity that we do not wish to tackle at this time. But using equations (81) and (82) and applying the result of our analysis of the reflecting polarizer for every spectral component hence calculated, we can get a better idea of the phenomenon considered here.

We have done this, and the result is shown in Figure 6, where we present the result of a simulation of the effect of a Gaussian beam on the width and shape of the resonance exhibited by a reflecting polarizer of the kind discussed in $\S 4.3$. The beam is converging with its waist situated some $10 \mathrm{~cm}$ "behind" the polarizer, and the integrated power (over a beam width) is measured some distance away from the assembly in the far field. Although there still remain some differences, this simulation shares a lot of the same features observed experimentally.

We believe that simulations like this one along with our earlier calculations provide convincing and compelling evidence for the importance of appropriately taking into account 
the nature of the incident radiation in the analysis of similar systems.

\section{CONCLUSION}

In this paper, a general solution for the analysis of polarizing grids was presented; it is valid for arbitrary angles of incidence and of grid rotation. With it and the scattering matrix representation that derives from it, basically any configuration or system of grids can be analyzed as long as some assumptions concerning the wire radius and spacing are respected $(\lambda>$ $40 a$ and $d>4 a$ ). This is not a severe restriction, as most grids currently available satisfy those conditions; we refer the reader to Chambers, Parker, \& Costley (1986) and Chambers, Costley, \& Parker (1988) for cases where a larger size of wire is needed. Our analysis also allowed us to define a set of optimum values for both the wire radius and spacing as specified by the following equations:

$$
\begin{aligned}
& a \simeq\left[\frac{\lambda^{5}}{\left(1-\alpha^{2}\right)^{4} \pi^{7} \sigma Z_{0}}\right]^{1 / 6}, \\
& d \simeq 2 \pi a .
\end{aligned}
$$

We provided an analysis of the effects that two types of random errors can have on the performance of a grid. It was shown that errors in the wire spacing were the most important and could have some impact on the amount of unwanted polarization transmitted through a grid. In that respect, our model was shown to be in good agreement with the experimental results of Shapiro \& Bloemhof (1990).

Comparisons with experimental data obtained in the calibration of a reflecting polarizer used at the OVRO were also presented, and predictions from our model are in good agreement with it. The only discrepancies appeared in the nature of a resonance, more precisely its width. But we have shown that it could be accounted for by including in the analysis a proper treatment of the effects of the nature of the incident radiation on the response of the polarizer.

We are grateful to J. B. Shapiro and E. E. Bloemhof for their permission to use their previously published experimental results. We wish to thank the staff of the Owens Valley Radio Observatory and O. P. Lay for numerous discussions and suggestions. The Owens Valley Radio Observatory is funded by the National Science Foundation under contract AST 96-13717 and the polarimetry project at OVRO through NASA grant NAG5-4462. The work of M. H. was supported in part by a grants from FCAR and the Département de Physique de l'Université de Montréal.

\section{APPENDIX A GENERALIZATION OF THE PRINCIPAL AXES}

Referring to equations (49) for the scattering matrix, one first realizes that such a symmetry in its components will always be seen when representing an arbitrary polarizing grid. Only the functions which define $R^{u u}, T^{u u}, \ldots$ will change. Further, when trying to reduce the scattering matrix to a form similar to equation (56), it is necessary to concentrate on only one of the two blocks (each appearing twice) present in equation (49). For example, if one diagonalizes the block composed of the reflection coefficients, then the transmission block is also diagonalized and vice versa. In obtaining the results that follow, we have worked with the reflection block appearing in the upper left and lower right of equation (49). We will now show that the orientation of the principal axes is determined by the symmetry of the grid and can be deduced using the formalism of group theory.

As seen by the incident wave, the grid has a symmetry which can be expressed by a representation of the point group $C_{2 v}$. The four coverings involved are the identity $(E)$, a rotation by $\pi$ about the $w$-axis $\left(C_{2}\right)$, a reflection $\left(\sigma_{v}\right)$ across a plane defined by the $w$-axis and an axis defined by the projection of the direction of the wires in the plane of the incident field, and finally another reflection $\left(\sigma_{v}^{\prime}\right)$ across a plane perpendicular to the previous one (and to the plane of the incident field). Upon studying the character table of this group (see Tinkham 1964, p. 325) and the effect of the above operations on the two possible states of linear polarization (along the $u$ - and $v$-axes), we find that only two nondegenerate irreducible representations $\left(B_{1}\right.$ and $B_{2}$ in Tinkham 1964 , p. 325) will be realized. For each of these there will exist one eigenvector, each corresponding to a given principal axis. These can be deduced by constructing the appropriate symmetry coordinates (Wilson, Decius, \& Cross 1955) which turn out to be the two principal axes $\boldsymbol{p}_{1}$ and $\boldsymbol{p}_{2}$ previously defined in $\S 3.3 .1$.

Since this result was obtained with the use of group theory, it is perfectly general and independent of any approximations that can be used in dealing with a polarizing grid. 


\section{APPENDIX B}

\section{LIST OF SYMBOLS}

$a$ : wire radius,

$\boldsymbol{A}_{s}$ : scattering vector potential,

$c$ : speed of light in free space,

$d$ : wire spacing,

$\boldsymbol{E}_{i}, \boldsymbol{E}_{s}, \boldsymbol{E}_{T}$ : incident, scattered and total electric field,

$F$ : constant $\left(=\pi \mu_{0} \omega a / 2\right)$,

$\boldsymbol{F}_{s}$ : scattering vector potential,

$h$ : grid-mirror separation (reflecting polarizer),

$\boldsymbol{H}_{s}$ : scattered magnetic field,

$H_{n}^{(2)}$ : Hankel function of the second kind of order $n$,

$J$ : current density vector,

$J_{n}$ : Bessel function of the first kind of order $n$,

$\boldsymbol{k}$ : wavevector of the incident wave $(|k|=k=2 \pi / \lambda)$,

$k^{\prime}:=k \sqrt{1-\alpha^{2}}$,

$\boldsymbol{K}$ : total surface current density vector,

$\overline{K^{x}}, \overline{K^{\theta}}$ : mean longitudinal and azimuthal surface current densities,

$\boldsymbol{p}_{1}, \boldsymbol{p}_{2}$ : principal axes of a grid,

$R^{x}, R^{y}, R^{z}$ : reflection coefficients in the system of coordinates of the grid (see Fig. 1),

$R^{u^{\prime}}, R^{v^{\prime}}$ : reflection coefficients in the system of coordinates of the laboratory (see Figs. 1 and 2),

$R_{\|}, R_{\perp}$ : reflection coefficients along the principal axes of a grid,

$R_{\mathrm{TE}}, R_{\mathrm{TM}}$ : transverse electric and transverse magnetic reflection coefficients of the mirror,

$S$ : scattering matrix,

$T^{x}, T^{y}, T^{z}$ : transmission coefficients in the system of coordinates of the grid (see Fig. 1),

$T^{u}, T^{v}$ : transmission coefficients in the system of coordinates of the laboratory (see Figs. 1 and 2),

$T_{\|}, T_{\perp}$ : transmission coefficients along the principal axes of a grid,

$W_{0}, z_{R}$ : beam waist and Rayleigh range of a circular Gaussian beam,

$Z$ : impedance matrix,

$Z_{0}$ : impedance of free space $\left(=\sqrt{\mu_{0} / \varepsilon_{0}}\right)$,

$Z_{p}, Z_{n}$ : grid impedance along the principal axes (as defined with the impedance matrix),

$Z_{s}$ : surface impedance of the wires $\left[=(1+j) \sqrt{\mu_{0} \omega / 2 \sigma}\right]$,

$Z_{\|}, Z_{\perp}$ : grid impedance along the principal axes (as defined with the scattering matrix),

$\alpha$ : projection of the normalized wavevector on the $x$-axis $\left[=\sin \left(\chi_{i}\right) \sin \left(\varphi_{g}\right)\right]$,

$\beta$ : projection of the normalized wavevector on the $y$-axis $\left[=\sin \left(\chi_{i}\right) \cos \left(\varphi_{g}\right)\right]$,

$\gamma$ : projection of the normalized wavevector on the $z$-axis $\left[=\cos \left(\chi_{i}\right)\right]$,

$\alpha^{\prime}, \beta^{\prime}, \gamma^{\prime}$ : projection of the normalized incident field on the $x, y$ and $z$-axes,

$\alpha^{\prime \prime}, \beta^{\prime \prime}$ : projection of the normalized incident field on the $u$ - and $v$-axes,

$\delta(x)$ : Dirac's delta distribution,

$\varepsilon_{0}, \mu_{0}$ : permittivity and permeability of free space,

$\eta, \xi$ : random errors in wire radius and spacing,

$\lambda$ : wavelength,

$\sigma$ : wire conductivity,

$\varphi_{g}, \chi_{i}$ : angle of grid rotation and angle of incidence,

$\Psi$ : Euler's constant $(\simeq 0.577215)$,

$\omega$ : angular frequency of radiation. 


\section{REFERENCES}

Akeson, R. L. 1997, Ph.D. thesis, Caltech

Akeson, R. L., Carlstrom, J. E., Phillips, J. A., \& Woody, D. P. 1996, ApJ, 456, L45

Balanis, C. A. 1989, Advanced Engineering Electromagnetics (New York: John Wiley \& sons), chap. 6

Bouche, D., Molinet, F., \& Mittra, R. 1997, Asymptotic Methods in Electromagnetics (Berlin: Springer), chap. 3

Chambers, W. G., Costley, A. E., \& Parker, T. J. 1988, Int. J. Infrared Millimter Waves, 9(2), 157

Chambers, W. G., Parker, T. J., \& Costley, A. E. 1986, Infrared Millimeter Waves, 16, 77

Collin, R. E. 1992, Foundations for Microwave Engineering (New York: McGraw-Hill)

Fowles, G. R. 1975, Introduction to Modern Optics (New York: Dover), 164

Jackson, J. D. 1962, Classical Electrodynamics (New York: Wiley)
Lamb, J. W. 1997, Modeling of Circular Polarizer Response, OVRO Internal Memo.

Larsen, T. 1962, IRE Transactions on Microwave Theory and Techniques, vol. 10 (New York: IRE), 191

Shapiro, J. B., \& Bloemhof, E. E. 1990, Int. J. Infrared Millimeter Waves, 11(8), 973

Tinkham, M. 1964, Group Theory and Quantum Mechanics (New York: McGraw-Hill)

van de Hulst, H. C. 1957, Light Scattering by Small Particles (New York: Dover)

Wait, J. R. 1954, Canadian J. Phys., 32, 571

$$
\text { 1955, Appl. Sci. Res., 4, B393 }
$$$$
\text { 1979, Electr. Lett., 15(20), } 659
$$

Wilson, E. B., Jr., Decius, J. C., \& Cross, P. C. 1955, Molecular Vibrations (New York: Dover) 